\title{
At the interface
}

\author{
Peter Prevelige \\ Professor, Department of Microbiology, University of Alabama at Birmingham
}

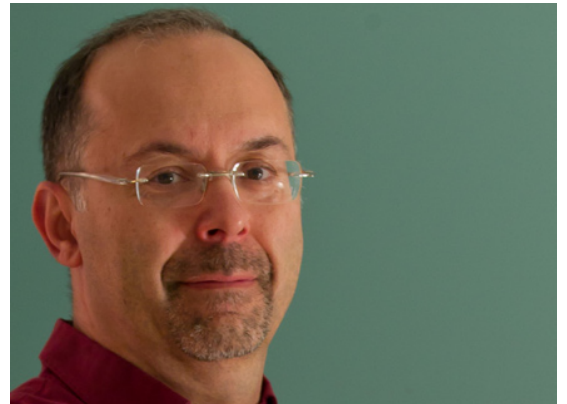

Peter Prevelige's research into viral capsid assembly and his contributions to the field of nanobiotechnology caught our attention. Curious to know more, BioTechniques contacted him to find out about the ambition, character, and motivation that led to his success.

How did you first become interested in biology and virology?

I was born in the mid-50s during the era of Sputnik. My mother was fascinated with the 'space race' and how science was glorified at the time, so I grew up running around museums in New York, New Jersey, and Connecticut. As a result, I decided to study biology in college and biochemistry in graduate school.

During graduate school at Brandeis University, I took a course in biological self-assembly taught by Don Caspar, one of the luminaries of structural virology. I was the only student in the course, so we would simply talk about how protein molecules come together to give shape, form, and dynamics to different biological systems, including viruses. My thesis work focused on histones, but this course sparked my interest in viral capsid assembly, so when an opportunity for postdoctoral studies presented itself at MIT with the virologist Jonathan King, I accepted.

What do you believe has been your most valuable scientific contribution?

Our most significant contribution has been the identification of an interface between the $\mathrm{N}$ - and C-terminal domains of the capsid protein of HIV that occurs during the transition from an immature non-infec- tious form to the infectious form. At the time, data suggested the need for self-interaction during HIV capsid maturation, but the precise mechanism couldn't be determined. The challenge with performing structural studies on $\mathrm{HIV}$ is that the particles are so irregular that it is very difficult to determine structures of mature or immature capsids using either cryoelectron microscopy or x-ray crystallography. We had recently become interested in hydrogen-deuterium exchange studies for characterizing protein-protein interactions and realized we could combine that approach with mass spectrometry to look at the structure of the HIV capsid. It seemed a perfect way to identify this potential interface. Since describing the interface, a number of small molecules designed to block that structural rearrangement have been developed for preclinical studies.

\section{What are you working on now?}

There are currently three related focuses in the laboratory. First, we are studying the assembly of viral capsids from protein subunits using the Phi29 bacteriophage with the goal of moving into single molecule studies. To this end, we have developed an in vitro assembly system and are currently putting fluorescent tags on individual protein subunits so we can watch them come together using FRET and TIRF microscopy approaches.

Our two other projects, which are collaborations with Trevor Douglas at Montana State University, involve using the bacteriophage P22 capsid as a scaffold or container. In one project, we are using the phage capsid as a scaffold to promote controlled mineralization for solar energy conversion, and in the other, we are developing capsids as a platform for biomedicine. We have become quite adept at loading cargo inside these particles, so now we are working to engineer the exterior surface to target particular disease tissues and thereby bring specific imaging reagents or therapeutics to needed locations. My group is working with a phage display system to find particles that target specific cardiovascular disease tissues while Trevor's lab is improving our approach to loading imaging reagents into the particles.

Solar energy seems to be a departure from your other research. How did you adapt your approach?

Understanding capsid assembly to position things precisely in space is common to the two fields, but in the case of solar energy, the chemistry is different and new to me, which is a challenge. Our idea is to use closed spherical viral particles as containers for chemical reactions. We add peptides to the inside of the capsid, either attached to the inner surface or hanging from it, that interact with inorganic materials to nucleate crystallization. Trevor and I actually have an additional collaboration with Arunava Gupta, an expert in materials science, who aids us in choosing materials. When these capsids are assembled correctly, we hope the crystals will absorb photons and split water; we have very recent data showing this is possible.

What is the most important open research question in your field?

What we currently lack is a clear definition of the interactions required for proper form determination at the molecular level. Proper assembly of capsids containing infectious nucleic acids occurs in a noisy cellular environment where there are many other proteins, nucleic acids, and components that could potentially get caught up in the process. So we need to discover at the molecular level how those interactions occur as well as the binding strengths between subunits and intermediates. This information is essential for targeting those steps for developing anti-viral therapies. Additionally, by understanding the design principles, we can better co-opt them for nanobiotechnology applications in the future.

Interviewed by Kristie Nybo, Ph.D. Image courtesy of Peter Prevelige. 楜 